\title{
Integrated palliative care in Europe: a qualitative systematic literature review of empirically-tested models in cancer and chronic disease
}

\author{
Naouma Siouta ${ }^{1 *}$, K. Van Beek ${ }^{1}$, M. E. van der Eerden ${ }^{2}$, N. Preston ${ }^{3}$, J. G. Hasselaar ${ }^{2}$, S. Hughes ${ }^{3}$, E. Garralda ${ }^{4,7}$,
} C. Centeno ${ }^{4,7}$, A. Csikos 5 , M. Groot ${ }^{2}$, L. Radbruch ${ }^{6}$, S. Payne ${ }^{3}$ and J. Menten ${ }^{1}$

\begin{abstract}
Background: Integrated Palliative Care (PC) strategies are often implemented following models, namely standardized designs that provide frameworks for the organization of care for people with a progressive life-threatening illness and/or for their (in)formal caregivers. The aim of this qualitative systematic review is to identify empirically-evaluated models of PC in cancer and chronic disease in Europe. Further, develop a generic framework that will consist of the basis for the design of future models for integrated PC in Europe.

Methods: Cochrane, PubMed, EMBASE, CINAHL, AMED, BNI, Web of Science, NHS Evidence. Five journals and references from included studies were hand-searched. Two reviewers screened the search results. Studies with adult patients with advanced cancer/chronic disease from 1995 to 2013 in Europe, in English, French, German, Dutch, Hungarian or Spanish were included. A narrative synthesis was used.

Results: 14 studies were included, 7 models for chronic disease, 4 for integrated care in oncology, 2 for both cancer and chronic disease and 2 for end-of-life pathways. The results show a strong agreement on the benefits of the involvement of a PC multidisciplinary team: better symptom control, less caregiver burden, improvement in continuity and coordination of care, fewer admissions, cost effectiveness and patients dying in their preferred place.

Conclusion: Based on our findings, a generic framework for integrated PC in cancer and chronic disease is proposed. This framework fosters integration of PC in the disease trajectory concurrently with treatment and identifies the importance of employing a PC-trained multidisciplinary team with a threefold focus: treatment, consulting and training.
\end{abstract}

Keywords: Delivery of health care, Integrated, Palliative care, Review, Systematic, Medical oncology, Chronic disease

\section{Background}

According to the World Health Organization (WHO), Palliative Care (PC) aims to improve the quality of life of patients and families who face life-threatening illness, by providing pain and symptom relief, spiritual and psychosocial support from diagnosis to end of life care and bereavement. Further, the WHO recommends that PC becomes an integral part of health care and that all

\footnotetext{
* Correspondence: naouma.siouta@gmail.com; naouma.siouta@kuleuven.com 'Department of Radiation-Oncology and Palliative Medicine, University Hospital Gasthuisberg, Herestraat 49, 3000 Leuven, Belgium

Full list of author information is available at the end of the article
}

patients affected by a life threatening disease should have access to PC services [1]. This statement is further supported by the European Association of Palliative Care [2] and is also in agreement with the guidelines of the European Council towards the European Union (EU) Member States [3].

This consensus in favour of integrating $\mathrm{PC}$ within regular treatment offered to patients with life-threatening disease is supported by a growing amount of evidence that indicates the effectiveness of $\mathrm{PC}$ on the improvement of the quality of life of these patients [4-8]. 
The implementation of integrated PC strategies is often based on models. Models of care are standardized designs that provide frameworks for the organization of care for people with a progressive life-threatening illness and/or for their (in)formal caregivers. As such, models determine the norms of PC practice and offer values and principles that professionals can use as guides and can thus provide important information for understanding integrated PC practices and evaluating their associated strengths and weaknesses.

To date, a univocally accepted model for PC delivery and integration does not exist, even if we confine ourselves to cancer or a major chronic disease. However, there are calls for developing generic PC models that will incorporate recent findings concerning the early identification of patients with needs for PC services and foster the integration of PC early in the care plan and throughout the disease trajectory [9-15].

In the present study, we perform a qualitative systematic review of the available literature for evidence-based models of integrated PC in Europe. We confine ourselves to studies that empirically measure the effectiveness of the corresponding models and employ a high-quality methodological rigour. Further, we examine the conformance of the included studies with respect to how the characteristics and requirements of integrated PC and strengths and weaknesses are documented. By incorporating the strengths and by rectifying the weaknesses, we propose a generic framework that aspires to demonstrate how to integrate PC both in cancer and chronic disease. This study is part of the European project InSup-C that focuses on integration of $\mathrm{PC}$ in advanced cancer and chronic disease in Europe (http://www.insup-c.eu/).

\section{Methods}

A unanimously agreed definition of integrated PC does not exist as such. For the needs of the present study, a novel definition was developed in the course of the InSup-C meetings by the PC experts and the authors. This definition combines all the aspects of integrated PC, identified by both experts and literature. Our working definition is: "Integrated palliative care involves bringing together administrative, organisational, clinical and service aspects in order to realise continuity of care between all actors involved in the care network of patients receiving palliative care. It aims to achieve quality of life and a well-supported dying process for the patient and the family in collaboration with all the care givers (paid and unpaid)".

It is important to note that this definition does not distinguish between timings that integration of $\mathrm{PC}$ can commence. Consequently, both early and end-of-life PC care can be part of this review.
Finally, this qualitative systematic review was conducted in Belgium in September of 2013.

\section{Selection criteria}

Eligible studies included those focusing on models of integrated PC for adult patients with cancer or another chronic disease (COPD, renal failure, heart failure, HIV, dementia or other types of neurological diseases), that are, in turn, consistent with the above-mentioned definitions of models and of integrated PC. Since our primary objective concerns the identification of evidence based models, only those studies that empirically assessed the effectiveness of these models and provided relevant data were considered eligible. In particular, we considered randomized controlled trials (RCTs), quasi experimental studies, cohort studies, controlled before-and-after studies, observational studies and pilot evaluation studies whereas we excluded theoretical studies, audits, opiniononly studies in clinical case reports, editorials and letter.

We confided ourselves to studies published from 01-011995 (based on the publication year of the Calman-Hine report which constitutes the first national cancer plan in Europe [16]) to 31-12-2013 in one of the following languages: English, French, German, Dutch, Hungarian and Spanish which are the languages that the authors were knowledgeable of.

A final eligibility criterion concerned the quality of the included studies with respect to the methodological rigor. In the present systematic review, only studies that that scored at least $60 \%$ (above 22/36) in the Hawker quality assessment scale were included in this systematic review; see Quality assessment section below.

\section{Search strategy}

The following databases were searched electronically: The Cochrane Central Register of Controlled Trials (CENTRAL), PubMed, EMBASE, CINAHL, AMED, BNI, Web of Science and NHS Evidence. The search in the databases was performed via the use of keywords, MESH terms and search terms as well as their permutations and combinations. The basic search terms and keywords that were used in PubMed and similarly in the other electronic databases are presented in Appendix. Validation of the search strategy was performed against five key papers [4-8].

Additionally, the following journals were hand-searched: BMJ Supportive \& Palliative Care, European Journal of Palliative Care, Journal of Pain and Symptom Management, Palliative Medicine and Medicina Paliativa. Citation tracking was also performed for the included studies.

Two different grey literature searches were performed. First, we identified and contacted experts in national scientific medical organizations in order to acquire additional information on existing models. Second, we 
carried out an electronic search in Google by utilizing a language-tailored strategy; more specifically, each country used relevant key-words and search terms translated into its corresponding language.

\section{Selection procedure}

In the first phase, two reviewers (NS \& KVB) screened all the search results on the basis of their title and their abstract. Non-English titles were screened and translated by two native speaker reviewers. The full texts of articles selected by both reviewers were sourced. Following standard practice, discrepancies were resolved by consensus.

\section{Data extraction}

Data were extracted from papers meeting the inclusion criteria using an extraction form built upon the one described in Hawker et.al. This extraction form was modified for the purposes of this study following consensus in the InSup-C project meetings [17]. For each included paper, data extraction was carried out by the first two authors independently for the cross-checked their results and reached consensus on discrepancies. Extracted variables were: first author's name, year of the study, country the study was conducted, design, quality assessment, description of the study model, outcome measures, results, focus of the model, setting, time frame of the model, disciplines represented and collaboration strategy.

\section{Quality assessment}

The methodological rigour of each included study was formally assessed by the numerical scoring system designed by Hawker et al. [17]. This scoring system is based on nine criteria that are evaluated with a fourpoint Likert scale: good (4) to very poor (1), thus yielding a maximum score of 36 . The nine criteria are: abstract/title, introduction/aims, methods/data, sampling, data analysis, ethics and bias, findings/results, transferability/generalizability, implications/usefulness. It is important to note that the criterion corresponding to methods takes into account the design of the study as well, e.g. RCT, observational study etc. It is on this premise that in the present systematic review we have included studies with different designs and not only RCTs. A detailed presentation of the Hawker tool is reported in Table 1. Our choice is also influenced by Oishi et al. and Rigby et al. who used the Hawker tool while conducting a PC-related systematic review $[18,19]$.

\section{Data synthesis}

The included studies were characterized by a substantial heterogeneity as studies with both clinical and methodological diversities were included. For this reason, a narrative synthesis was favoured over a meta-analysis and data are reported in tables; hence the qualitative nature of the review mentioned above. This heterogeneity has also affected the presentation of the results. Comparative analysis was only possible by using a specific number of categories (design, study populations, assessments and outcomes) that will be presented in the following sections.

\section{Results}

The database search returned 28,274 hits, excluding duplicates. From the studies that were screened based on abstract/title, 1989 were found to be eligible for full-text screening. Upon full text screening, 491 studies qualified for further assessment of their eligibility. Finally, 14 studies were found to comply with all the selection criteria (see relevant section above). The grey literature search did not add any empirical studies. A flow diagram of the selection procedure and the results is shown in Fig. 1.

From the 14 studies, 7 evaluated models about chronic disease (dementia, multiple sclerosis, chronic heart failure, HIV/AIDS and advanced chronic disease in general), 4 about integrated care in oncology, 3 about cancer and chronic disease (end-of-life patients). The majority of the included studies were from the UK (6 out of 14), 2 were from Spain, 2 from The Netherlands, and one each from Italy, Germany, Norway and France. The preponderance of UK studies is clear in chronic disease where all 6 included studies were from this nation.

The 14 included studies were built upon quite different designs (Table 2) [20-33]. More specifically, there were 6 RCTs, 2 observational studies, 2 cohort studies, 2 pilot evaluation studies, 1 uncontrolled before-and-after studies and 1 quasi-experimental study.

Table 2 provides information concerning the quality of the included studies, as measured via the Hawker et al. tool. Two (2) studies [29, 30] scored a perfect 36 while 3 other studies scored 33 or 34 . The majority of the remaining studies scored in the range between 23 and 31 . Table 2 includes detailed information concerning the models of the included studies, their outcome measures and the results/effectiveness of the corresponding models. From this table, we can infer that although RCTs tend to score higher and observational studies lower, other designs perform reasonably well. The latter indicates that such studies have a sound basis, despite the fact that they do not employ an RCT for their assessment.

Even though some of the models bear similarities, (see Table 3 Screening), differences associated with the care setting and the compositions of the team render further grouping and comparison difficult. Upon detailed examination, a common ground for comparison was found in the following categories: focus of intervention, the setting, the timing of the intervention in the disease 
Table 1 Hawker tool description

\begin{tabular}{|c|c|}
\hline \multicolumn{2}{|c|}{ 1. Abstract and title: Did they provide a clear description of the study? } \\
\hline Good & Structured abstract with full information and clear title. \\
\hline Fair & Abstract with most of the information. \\
\hline Poor & Inadequate abstract. \\
\hline Very poor & No abstract. \\
\hline
\end{tabular}

2. Introduction and aims: Was there a good background and clear statement of the aims of the research?
Good Full but concise background to discussion/study containing up-todate literature review and highlighting gaps in knowledge.

Clear statement of aim AND objectives including research questions.

Fair Some background and literature review.

Research questions outlined.

Poor Some background but no aim/objectives/questions, OR Aims/objectives but inadequate background.

Very poor No mention of aims/objectives.

No background or literature review.

3. Method and data: Is the method appropriate and clearly explained?

Good Method is appropriate and described clearly (e.g., questionnaires included). Clear details of the data collection and recording.

Fair Method appropriate, description could be better.

Data described.

Poor Questionable whether method is appropriate.

Method described inadequately.

Little description of data.

Very poor No mention of method, AND/OR

Method inappropriate, AND/OR

No details of data.

4. Sampling: Was the sampling strategy appropriate to address the aims?

Good Details (age/gender/race/context) of who was studied and how they were recruited.

Why this group was targeted.

The sample size was justified for the study.

Response rates shown and explained.

Fair Sample size justified.

Most information given, but some missing.

Poor Sampling mentioned but few descriptive details.

Very poor No details of sample.

5. Data analysis: Was the description of the data analysis sufficiently rigorous?

Good Clear description of how analysis was done.

Qualitative studies: Description of how themes derived/respondent validation or triangulation.

Quantitative studies: Reasons for tests selected hypothesis driven/numbers add up/statistical significance discussed.
Table 1 Hawker tool description (Continued)

\begin{tabular}{|c|c|}
\hline \multirow[t]{2}{*}{ Fair } & Qualitative: Descriptive discussion of analysis. \\
\hline & Quantitative. \\
\hline Poor & Minimal details about analysis. \\
\hline Very poor & No discussion of analysis. \\
\hline \multicolumn{2}{|c|}{$\begin{array}{l}\text { Ethics and bias: Have ethical issues been addressed, and } \\
\text { what has necessary ethical approval gained? Has the } \\
\text { relationship between researchers and participants been } \\
\text { adequately considered? }\end{array}$} \\
\hline \multirow[t]{2}{*}{ Good } & $\begin{array}{l}\text { Ethics: Where necessary issues of confidentiality, } \\
\text { sensitivity, and consent were addressed. }\end{array}$ \\
\hline & $\begin{array}{l}\text { Bias: Researcher was reflexive and/or aware of own } \\
\text { bias. }\end{array}$ \\
\hline Fair & $\begin{array}{l}\text { Lip service was paid to above (i.e., these issues were } \\
\text { acknowledged). }\end{array}$ \\
\hline Poor & Brief mention of issues. \\
\hline Very poor & No mention of issues. \\
\hline \multicolumn{2}{|c|}{ Results: Is there a clear statement of the findings? } \\
\hline \multirow[t]{4}{*}{ Good } & $\begin{array}{l}\text { Findings explicit, easy to understand, and in logical } \\
\text { progression. }\end{array}$ \\
\hline & Tables, if present, are explained in text. \\
\hline & Results relate directly to aims. \\
\hline & Sufficient data are presented to support findings. \\
\hline \multirow[t]{2}{*}{ Fair } & $\begin{array}{l}\text { Findings mentioned but more explanation could } \\
\text { be given. }\end{array}$ \\
\hline & Data presented relate directly to results. \\
\hline Poor & $\begin{array}{l}\text { Findings presented haphazardly, not explained, } \\
\text { and do not progress logically from results. }\end{array}$ \\
\hline Very poor & Findings not mentioned or do not relate to aims. \\
\hline \multicolumn{2}{|c|}{$\begin{array}{l}\text { Transferability or generalizability: Are the findings of this study } \\
\text { transferable (generalizable) to a wider population? }\end{array}$} \\
\hline Good & $\begin{array}{l}\text { Context and setting of the study is described } \\
\text { sufficiently to allow comparison with other } \\
\text { contexts and settings, plus high score in } \\
\text { Question } 4 \text { (sampling). }\end{array}$ \\
\hline Fair & $\begin{array}{l}\text { Some context and setting described, but more } \\
\text { needed to replicate or compare the study with } \\
\text { others, PLUS fair score or higher in Question } 4 \text {. }\end{array}$ \\
\hline Poor & Minimal description of context/setting. \\
\hline Very poor & No description of context/setting. \\
\hline \multicolumn{2}{|c|}{$\begin{array}{l}\text { Implications and usefulness: How important are these findings to } \\
\text { policy and practice? }\end{array}$} \\
\hline \multirow[t]{3}{*}{ Good } & $\begin{array}{l}\text { Contributes something new and/or different in } \\
\text { terms of understanding/insight or perspective. }\end{array}$ \\
\hline & Suggests ideas for further research. \\
\hline & Suggests implications for policy and/or practice. \\
\hline Fair & Two of the above (state what is missing in comments). \\
\hline Poor & Only one of the above. \\
\hline Very poor & None of the above. \\
\hline
\end{tabular}




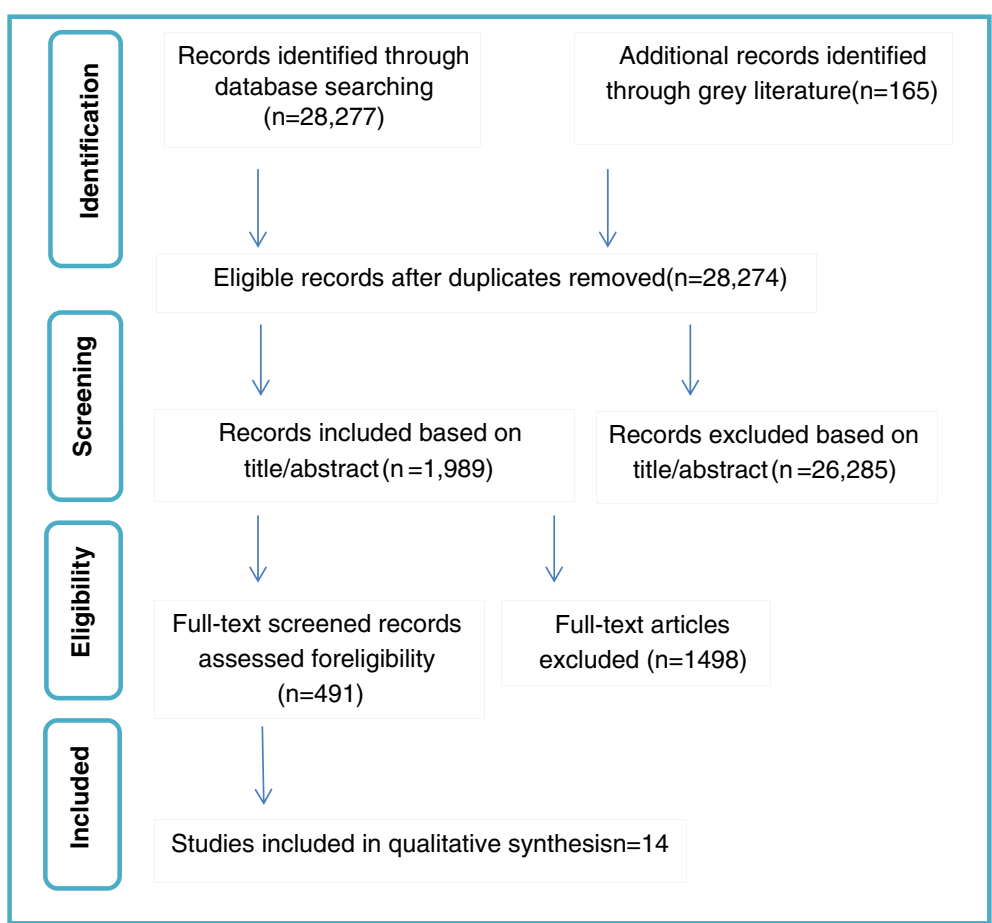

Fig. 1 Flow diagram of study selection procedure

trajectory, the collaboration strategy and the effectiveness of the models. In the following section, we confine the presentation of the results to these categories.

\section{Focus of intervention}

All the included studies placed their focus on symptom treatment. In particular, the objective of these interventions was to improve physical symptoms (dyspnoea, pain, constipation, nausea, vomiting, diarrhoea), emotional symptoms (agitation, confusion, fear, delirium) and to a lesser extent cognitive and social functioning [20, 23, 28-31]. Nine studies focused on consulting about end-of-life care decisions, advance care planning, advice for coping with life-threatening disease and referrals to specific care-settings. Five studies focused on the training of the nurses and physicians involved in the interventions. The various training programmes included training of the staff to support patients over the telephone [21], education on the symptoms of the disease [28] and more general educational programmes for community professionals about palliative care services $[20,24,28]$.

\section{Setting}

The implementation of the models has taken place in a variety of settings. In fact, most studies took place in more than one setting with the majority focusing on inpatient (11/14) and home care (9/11).

\section{Timing of PC initiation}

As regards the timing of the iniation of $\mathrm{PC}$, three types of studies were identified; those focusing on end-of-life (11), on concurrent care setting (where PC care is provided alongside regular treatment)(3) and on both (4). For the end-of-life setting, included patients typically were expected to die within a range spanning from a few weeks (2 weeks [26]) to few months (e.g. 2-9 months [20]). For the concurrent care setting, eligible patients were referred by clinicians as potentially benefiting from PC assessments [29, 30]. Finally, for the combined setting, various eligibility categories were identified. For example, in [23] all patients with malignant disease independent of the disease trajectory were eligible.

\section{Composition of team}

Three different team compositions were identified from the included studies. The first type refers to teams comprising medical and nursing staff; 2 studies [23, 25]. The second type concerns multidisciplinary teams comprising different professions: general practitioners (GPs), medical specialists, nurses and specialist nurses, psychiatrist and psychologists, health coordinators, administrative assistants, social workers, ambulance services, dieticians and rarely occupational therapists and speech therapists; 4 studies $[21,26,33]$. The third type corresponds to multidisciplinary teams that additionally involve PC experts. Besides being multidisciplinary, these 
Table 2 Characteristics of the included studies according to different disease categories

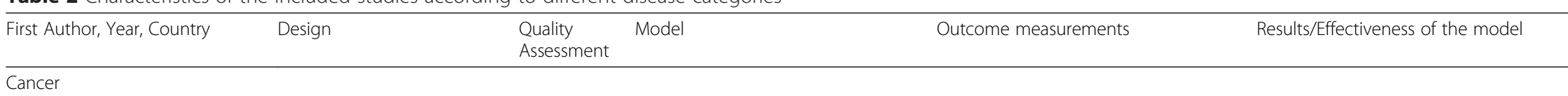

Jordhøy et al. 2001

(Norway) [20]

Cluster Randomised Trial

Quasi-experimental

Smeenk et al. 1998

(The Netherlands) [21]

study

Colombet et al. 2012,

(France) [22]

nested in a cohort
Case series study

Impact of oncologist's awareness

of $P C$ clinical intervention of $P C T^{*}$

and timing, multidisciplinary

decision-making.
Indicators: location of death, month of life, chemotherapy administration in last 14 days of life.

elease of pain, respiratory

Integration of PC into regular internal ward services in general hospital.

HRQL*: physical, emotional

functioning, pain, psychological distress. Place of death, hospital utilisation.

Re-hospitalization, QoL*, home death

distress, dying in hospital, length of hospital stay.

LCP* for cancer patients dying in hospital medical wards.

Effect of the LCP* on 3 health care settings (hospital, nursing home, home). of bereaved family member; communication between ward staff and GPs* (VOICES)*

Comparison of level of (EORTC QLQ-C30* questionnaire)
There was no evidence of any impact on the patients' $\mathrm{HROL}^{*}$. There was no tendency in favour of any treatment group on the main outcomes in assessments that were made within 3 months before death.

Patients in the intervention group underwent significantly less rehospitalization during the terminal phase of their illness; the intervention contributed significantly positive to the patients physical QoL; A higher, not significant percentage died at home.

58 patients died at home, 45 in an ICU or ER, and 253 in an acute care hospital; 185 patients visited the $\mathrm{ER}^{*}$ in last month of life and 75 received chemotherapy in last 14 days of life. OPM* independently decreases the odds of receiving decreases the odds of receiving chemotherapy in last 14 days of life. on indicators, whereas OPM* ${ }^{*}$ reduced the odds of persistent chemotherapy in the last 14 days of life. Decision-making with oncologists and the $\mathrm{PC}^{*}$ team is the most critical parameter for improving EoLC*

It is possible to integrate $\mathrm{PC}$ into a regular internal medical ward, with positive impact on recorded outcome measures

Quality of EoLC* from perspective documentation, symptom burden and aspects of communication (VOICES)* before and after introduction of LCP*.
Aspects of quality of EoLC* improved (emotional, spiritual needs, self-efficacy); slight improvement in communication, no significant improvement in symptom control.

\section{introduction of $L C P^{*}$ increased}

documentation, decreased symptom burden. 
Table $\mathbf{2}$ Characteristics of the included studies according to different disease categories (Continued)

Malignant and Non-malignant Disease
Grande et al. 2000, (UK) [26] Randomised controlled

Vicente et al. 2010, (Spain) [27] Retrospective and prospective cohort study

Dementia

Sampson et al. 2011, (UK) [28] Randomised controlled

Multiple Scherosis

Higginson et al. 2009, (UK) [29] Randomised controlled trial

Edmonds et al. 2010, (UK) [30] Randomised controlled trial

\section{HIV/AIDS}

Koffman et al. 1996, (UK) [31] A descriptive pilot evaluation
Cambridge Hospital at Home for palliative care $(\mathrm{CHAH})$

Influence of the Integrated Plan of $\mathrm{PC}^{*}$ of the Autonomous Community of Madrid in the medical activity of a hospital based $P C^{*}$ unit.

Pilot implementation of the assessment of PC needs of patients with severe dementia and discussion with principal carers to improve EOLC*.

Evaluation of cost-

effectiveness of a new PC service for people with $\mathrm{MS}^{*}$.

Evaluation of a novel PC service.

Pilot evaluation of hospice at home service for patients with advanced HIV/AIDS; 24-h terminal care.
Symptom control, adequacy of care, kelihood of remaining at home in their final 2 weeks, GP visits.

Improvement in continuity of care, coordination amongst assistant bodies, increase in mean stay at the $\mathrm{PCU}^{*}$, increase in number of home deaths, etc.

Kessler Distress Scale, EQ-5D* Decision Conflicts Scale, Decision Satisfaction Inventory, State Anger Scale, Life Satisfaction Scale Satisfaction with EoLC*, Advanced Dementia Scale (FAST Scale); Pain and distress (the Abbey pain scale, the PACSLAC and the Doloplus), delirium (Confussion Assessment Method),

Use of services, patient symptom (UNDS*, EDSS* and POS-8*), other outcomes, caregiver burden (ZB|*).

MS Impact Scale, POS-8*, ZB'* Modified Lawton positivity questionnaire.

STAS*, evaluating pain control, other symptom control, patient/ family anxiety, patient/family insight and communication betwee patient and family, between professionals, between professionals and patient and family.
CHAH appeared to be associated with better quality home care.

PC home care improves continuity in care of patients. Transfers to intermediate stay care centers and deaths at home increased. Median stay at the $\mathrm{PCU}^{*}$ decreased.

General unwillingness to address EoL* issues. All carers were keen to receive more information about EoL* issues in dementia, found discussions very helpful. Participation of clinical MD* team facilitated integration of intervention with the clinical service.

Short-term PCT* was found to be costeffective, reducing inpatient and

community costs, caregiver burden and possibly patient pain

MS patients who received $\mathrm{PC}^{*}$ service had improvements in 5 key symptoms (pain, nausea, vomiting, mouth problems and sleeping difficulties) on the POS and improved informal caregiver wellbeing.

$80 \%$ died at home; STAS* showed improvements in items 'other symptoms control' and family insight. 
Table $\mathbf{2}$ Characteristics of the included studies according to different disease categories (Continued)

Chronic Heart Failure

\begin{tabular}{|c|c|c|c|c|}
\hline Pattenden et al. 2012, (UK) [32] & $\begin{array}{l}\text { Non-randomised pilot } \\
\text { evaluation }\end{array}$ & 30 & $\begin{array}{l}\text { Collaborative } \mathrm{PC}^{*} \text { for advanced } \\
\text { heart failure. }\end{array}$ & $\begin{array}{l}\text { Death in preferred place of care; } \\
\text { hospital admissions averted; costs } \\
\text { of medical procedures, inpatient } \\
\text { care and directs costs of intervention }\end{array}$ \\
\hline
\end{tabular}

Advanced Chronic Disease

$\begin{array}{ll}\text { Navarro et al., 2011, (Spain) [33] } & \begin{array}{l}\text { Observational, retrospective } \\ \text { and descriptive study }\end{array}\end{array}$

EoLC* of advanced chronic non-

cancer patients identified by

multidimensional evaluation

and interdisciplinary teamwork

in a medium and long term

hospital.

This pilot study provides tentative evidence that a collaborative homebased $\mathrm{PC}^{*}$ service for patients with advanced CHF may increase the
likelihood of death in place of choice and reduce inpatient admissions.

General data, terminal criteria, diagnostic and prognostic

information, development of advance directives, limiting levels of effort care, times from admission, risk of complicated bereavement.

Identification of advanced chronic noncancer patients and their needs by interdisciplinary teamwork enabled

indication for PC soon after admission

and ensured appropriate care during their stay.

Table 2 describes studies by each disease category according to author, year, country, design, quality assessment score, model, outcome measures and results/findings

Abbreviations: $P C$ palliative care, QoL quality of life, $P C T$ palliative care team, OPM onco-palliative meeting, EoL end of life, VOICES Views of Informal Carers - Evaluation of Services questionnaire, ER emergency room, MDT multidisciplinary team, QoC quality of care, GSFCH Gold Standards Framework in Care Homes, LCP Liverpool Care Pathway, PCU palliative care unit, UNDS United Kingdom Neurological Disability Scale, EDSS

Expanded Disability Status Scale, POS-8 Palliative Care Outcome Scale, ZBI Zarit Carer Burden Inventory, ESH Hospital Support Team, EQ-5D EuroQOL five dimensions questionnaire, EoLC end of life care, MD medical doctor, MS multiple sclerosis, STAS Support Team Assessment Schedule, ICU intensive care unit, CKD Chronic Kidney Disease 
Table $\mathbf{3}$ Characteristics of the models

\begin{tabular}{|c|c|c|c|c|c|}
\hline First author & Focus of the model & Setting & Time frame & Disciplines represented & Collaboration strategy \\
\hline \multicolumn{6}{|l|}{ Cancer } \\
\hline Jordhoy et al. [20] & treating, training, consulting & $\begin{array}{l}\text { Hospital, GP's, nursing homes, } \\
\text { home care }\end{array}$ & end of life & $\begin{array}{l}\text { GP, community nurse, consultant } \\
\text { nurse, physician from PMU. }\end{array}$ & Model-responsible team meetings ${ }^{a}$ \\
\hline Smeenk et al. [21] & treating, training, consulting & Hospital, primary care team & concurrent, end of life & $\begin{array}{l}\text { Specialist nurse coordinator, } \\
\text { oncology ward nurses + medical } \\
\text { specialist, transmural home team } \\
\text { with nurses from hospital + day care. }\end{array}$ & $\begin{array}{l}\text { Model-responsible team meetings, } \\
\text { protocol }\end{array}$ \\
\hline Colombet et al. [22] & treating, consulting & Hospital & concurrent, end of life & $\begin{array}{l}15 \text { referral physicians: oncologists } \\
\text { who prescribe chemotherapy, of } \\
\text { whom } 2 \text { have been trained in PC } \\
\text { fundamentals. MDT: PCT and } \\
\text { oncology staff. PCT: PC specialists, } \\
\text { nurses, secretary assistant, } \\
\text { psychologist. Oncology staff: } \\
\text { physicians, nurses, head nurses, } \\
\text { social workers, psychologists, } \\
\text { secretaries. }\end{array}$ & $\begin{array}{l}\text { Model-responsible team + additional } \\
\text { experts meeting }\end{array}$ \\
\hline Schreml, et al. [23] & treating, training, consulting & Hospital & concurrent, end of life & Physicians and nurses. & Model-responsible team meetings \\
\hline \multicolumn{6}{|c|}{ End-of-life (Liverpool Care Pathway) } \\
\hline \multirow[t]{2}{*}{ Constantini et al. [24] } & treating, training & Hospital & end of life & $\begin{array}{l}\text { PCT: } 2 \text { physicians, } 3 \text { nurses, } 2 \\
\text { psychologists. }\end{array}$ & protocol \\
\hline & & & & $\begin{array}{l}\text { LCP training: nurses and physicians } \\
\text { of the hospital wards. }\end{array}$ & \\
\hline Veerbeek et al. [25] & treating & $\begin{array}{l}\text { Hospital, nursing home and } \\
\text { home }\end{array}$ & end of life & Physicians and nurses. & $\begin{array}{l}\text { Model-responsible team meetings, } \\
\text { protocol }\end{array}$ \\
\hline \multicolumn{6}{|c|}{ Malignant and Non-malignant Disease } \\
\hline Grande et al. [26] & treating & Home & end of life & $\begin{array}{l}\text { Six qualified nurses, } 2 \text { nursing } \\
\text { auxiliaries, CHAH coordinator, } \\
\text { agency nursing care. }\end{array}$ & Model-responsible team meetings \\
\hline Vicente et al.[27] & treating & Hospital, home & end of life & $\begin{array}{l}\text { PC home team as an MDT } \\
\text { comprised by physicians, nurses, } \\
\text { nurse assistants, and administrative } \\
\text { assistants, social workes, psychologists. }\end{array}$ & Model-responsible team meetings \\
\hline \multicolumn{6}{|l|}{ Dementia } \\
\hline Sampson et al. [28] & treating, training, consulting & Hospital, home & end of life & $\begin{array}{l}\text { Senior nurse experienced in dementia } \\
\text { and trained in PC; clinical MDT. }\end{array}$ & Model-responsible team meetings \\
\hline \multicolumn{6}{|l|}{ Multiple Sclerosis } \\
\hline Higginson et al. [29] & treating, consulting & $\begin{array}{l}\text { Home, hospital outpatient } \\
\text { clinic, care homes, hospital }\end{array}$ & concurrent & $\begin{array}{l}\text { Part-time PC medicine consultant, } 1 \\
\text { part-time clinical nurse specialist, } 1 \\
\text { administrator, } 1 \text { psychosocial worker. }\end{array}$ & Model-responsible team meetings \\
\hline
\end{tabular}


Table 3 Characteristics of the models (Continued)

\begin{tabular}{|c|c|c|c|c|c|}
\hline Edmonds et al. [30] & treating, consulting & $\begin{array}{l}\text { Home, hospital outpatient } \\
\text { clinics, care homes, hospital }\end{array}$ & concurrent & $\begin{array}{l}\text { Part-time consultant in PC Medicine } \\
\text { with specialist interest in neurological } \\
\text { conditions, part-time clinical nurse } \\
\text { specialist, full time administrator. }\end{array}$ & $\begin{array}{l}\text { Model-responsible team + additional } \\
\text { experts meeting }\end{array}$ \\
\hline \multicolumn{6}{|l|}{ HIV/AIDS } \\
\hline Koffman et al. [31] & treating, consulting & Hospice, home & end of life & $\begin{array}{l}\text { Nurses trained in PC; bank nurses for } \\
\text { night-sitting; } 2 \text { PC medicine consultants. }\end{array}$ & $\begin{array}{l}\text { Model-responsible team + additional } \\
\text { experts meeting }\end{array}$ \\
\hline \multicolumn{6}{|l|}{ Chronic Heart Failure } \\
\hline Pattenden et al. [32] & treating, consulting & $\begin{array}{l}\text { Homes, hospice, 'care of } \\
\text { the elderly' wards }\end{array}$ & concurrent, end of life & $\begin{array}{l}\text { Heart Failure nurse specialists, MCN } \\
\text { nurses, MCN health care assistants, } \\
\text { cardiology, 'care for the elderly' } \\
\text { consultants, district nurses, GPs. }\end{array}$ & $\begin{array}{l}\text { Model-responsible team }+ \text { additional } \\
\text { experts meeting, protocol }\end{array}$ \\
\hline \multicolumn{6}{|c|}{ Advanced Chronic Disease } \\
\hline Navarro et al. [33] & treating & Hospital & concurrent & $\begin{array}{l}\text { The MDT consists of physicians, head } \\
\text { nurse, ward nurses, auxiliary nurses, } \\
\text { collaborating with a dietician, } \\
\text { psychologist, social worker, } \\
\text { rehabilitation physician, physiotherapist, } \\
\text { occupational therapist and speech } \\
\text { therapist. }\end{array}$ & $\begin{array}{l}\text { Model-responsible team + additional } \\
\text { experts meeting }\end{array}$ \\
\hline
\end{tabular}

Table 3 describes five characteristics of the included studies: the focus of the model, the setting, the time frame of the model, the functions represented, and collaboration strategy involved

$P C$ palliative care, GP general practitioner, PMU palliative medicine unit, $P C T$ palliative care team, MDT multidisciplinary team, LCP Liverpool Care Pathway, MNC Marie Curie Cancer Care, CHAH Cambridge hospital at home service, ESAD Home Care Support Team, MDM multidisciplinary meetings

ameetings of the team that is involved in the implementation of the model

meetings between the team responsible for the implementation of the model and other disciplines involved in the treatment of the patient 
teams also have PC physicians, PC nurses and PC psychologists; 8 studies [22, 24, 31].

\section{Collaboration strategy}

The collaboration strategy refers to the ways that the represented disciplines cooperate and assess emerging issues. As shown in Table 3, the included studies employ three different collaboration strategies and their combinations: i) meetings of the team that is involved in the implementation of the model (model-responsible team) [20, 21, 23-28], ii) meetings between the model-responsible team together with experts from other disciplines involved in the treatment of the patient [22, $29,31-33]$, iii) utilization of predefined protocols [21, $24,25,32]$. It is important to note that the collaboration is not necessarily related to the composition of the team. For example, a model-responsible team may be multi-disciplinary but still base its decisions on meetings where additional disciplines are represented. In other words, the principal difference between (i) and (ii) is the participation of experts that are not directly related to the implementation of the model in question on a frequent basis.

\section{Effectiveness of the model}

As regards effectiveness, all but one study [20], showed positive outcomes in terms of better symptom control and better quality of life, better communication between personnel, patients and caregiver, more deaths at the patients' homes and more cost-effective care. However, this result should be interpreted with care given the variability in the design of the interventions, some of which were not RCTs and therefore of lower quality.

Importantly, even within RCTs, that constitute the most robust design, further comparisons between the results are cumbersome to perform. This is because discrepancies are observed both in the outcome measures and in the tools employed for their assessment. For example, as regards the evaluation of symptom management, some of the studies used the European Organization for Research and Treatment of Cancer guidelines (EORTC QLQ-C30) questionnaire [24, 25, 34], whereas others employed the United Kingdom Neurological Disability Scale (UNDS) [35], the Expanded Disability Status Scale (EDSS) [36], the Palliative Care Outcome Scale (POS-8) [37] and the Support Team Assessment Schedule (STAS) [29-31, 38] or the Abbey pain scale [39], the PACSLAC [40] and the Doloplus [28, 41]. Also, communication aspects were assessed via the Views of Informal Carers-Evaluation of Services (VOICES) [42] tool [24, 25]. Further, caregivers' burden was measured with the help of the Zarit Carer Burden Inventory (ZBI) [43], [29, 30].
Finally, stressful events were assessed via the Impact of Event Scale (IES) questionnaire [44].

\section{Discussion}

We reviewed existing literature on evidence-based models for integrated PC in patients with cancer or chronic disease in Europe. Since the focus of the present study was on the effectiveness of existing models, only specific designs were considered as eligible e.g. RCTs, cohort studies, etc. whereas others, such as theoretical studies, audits, etc., were explicitly excluded. In addition, in order to exclude empirical studies that were conducted with low-quality standards, the numerical tool of Hawker et al. [17] was employed for the assessment of the methodological rigour. In this respect, only those studies that scored at least $60 \%$ in this tool were eligible.

The database search resulted in 14 empirical studies that fulfilled the inclusion criteria. According to our findings, there are only a few models of integrated PC in Europe whilst the geographic distribution of the origin of these interventions is imbalanced, with 6 out of 14 studies coming from the UK.

Only 6 out the 14 studies were RCTs whilst the remaining employed different designs, e.g. observational studies. However, as mentioned above, studies employing a different design for their assessment scored highly on Hawker's tool as well. Consequently, such non-RCT studies can provide valuable insight into the strengths and weaknesses of extant studies that has to be taken into consideration if a complete description of the current state-of-the-art is targeted, as is the case of the present study.

Even though the included studies employ different outcome measures and a direct comparison is not possible, the effectiveness of the models (as assessed by the outcome measures) was confirmed in 13 out of 14 studies. In particular, as mentioned above, the positive impact typically involves better symptom control, higher quality of life, less caregiver burden, improvement in continuity and coordination of care, fewer hospital admissions, patients dying more frequently at home, and improved cost-effectiveness. Even though only 6 out of 14 studies were RCTs, and thus the results of the assessments for the remaining studies should be treated with care, the positive outcomes still add to the growing bulk of evidence corroborating the effectiveness of both usual and integrated PC on the improvement of the quality of life of patients with life-threatening disease [4-8].

We now shift to the development of a framework of integrated PC that could serve as a first step towards the development of a generic model for both malignant and non-malignant disease. Although the included studies suggest practices that are in concordance with existing 
recommendations and empirical findings, no study presents an intervention that is optimal in all aspects. Consequently, the generic framework that we propose is based on a selective combination of the strengths of the included interventions alongside a critical evaluation of their weaknesses and shortcomings. First, we present the aspects of the generic framework and subsequently we rationalize our choice and motivations.

This generic framework consists of the following aspects.

- Focus of intervention: The focus of intervention will be placed on symptom treatment, consulting of patients/family and training of the personnel.

- Setting: The design of the framework is such that it can be applied to every care setting.

- Timing of intervention: The intervention can be initiated throughout the disease trajectory either concurrently or in the end-of-life.

- Composition of team: The framework requires a multidisciplinary team with members that are trained in the delivery of PC. This team can consist of GPs, physician specialists, nurses and specialist nurses, psychologists, social workers and administrative assistants.

- Collaboration strategy: The collaboration strategy, which refers to the ways that the represented disciplines cooperate and assess emerging issues, should be based on the involvement of the multidisciplinary team and its meetings and the utilization of protocols.

The proposed framework has a threefold focus: treatment, consulting and training. In our context of integrated $\mathrm{PC}$, treatment corresponds to the alleviation of the physical (dyspnoea, pain, constipation, nausea, vomiting, diarrhoea) and psychological symptoms (agitation, confusion, fear, delirium). On the other hand, consulting refers to discussions and communications over treatment options and at a later stage to end-of-life care decisions. Moreover wishes, desires and needs of the patients can be taken into account as part of this advance care planning. Training involves the education of nurses, physicians and other related health care providers involved in the interventions. Training programmes can be very diverse, however, their principal focus should be on PC, its benefits and its provision.

The benefits of focusing on treatment are apparent, however, the benefits of the remaining two aspects are also well documented. As regards consulting, PC typically involves difficult medical and ethical decision making [4-8] for patients and their (in)formal caregivers. Importantly, the same studies assert that the burden associated with this decision making can be ameliorated with the assistance of trained PC staff through systematic consulting and informative discussions.

By contrast, regarding training, we first note that the generic framework should be utilized with extreme caution because these suggestions may need to be tailored to the specific situation. In other words, this framework pertains to assist PC teams in their planning and implementation of PC strategies and not to provide black-box solutions. In this respect, it should be highlighted that the improper application of generic frameworks, and in fact of every integrated PC framework, can jeopardize the effectiveness of the employed PC practices. A notable example is the case of the Liverpool Care Pathway (LCP) [45], which has been sometimes erroneously employed, leading to poor outcomes and has in turn been used as an excuse for poor quality care [46, 47]. Public outcry from the poor implementation of the LCP led the UK government to stop the use of the LCP.

Recent studies explicitly state that there is a correlation between the successful implementation of the LCP and the training of the staff [48]. Importantly, this constitutes a characteristic of integrated PC in general [49-51]. For this reason, future efforts should explicitly foster the educational aspect of the professional members.

The choice of the timing of the intervention and thus the referral criteria is a contentious topic in the literature of PC. Historically, PC studies focusing on cancer opted for an end-of-life time frame based on the premise that once cancer reaches the terminal phase, the disease trajectory declines rapidly so that end-of-life prognoses can be fairly accurate [52, 53]; still, prognosis in cancer is frequently over-optimistic [54]. However, early integrated PC is increasingly supported by various studies, because it has been documented to improve quality of life in the last days and increase survival [4].

Moreover, end-of-life prognosis for non-malignant disease is problematic. For example, the typical trajectory of Chronic Heart Failure (CHF) consists of acute crises or exacerbations followed by elongated periods of stability. At the same time, CHF patients are in high risk of sudden death [55]. Consequently, for non-malignant disease, an end-of-life frame is cumbersome to realise and a concurrent time frame of the initiation of $\mathrm{PC}$ is deemed more appropriate [56]. On the other hand, there is a growing number of studies supporting the decoupling of the initiation of PC from the traditional end-oflife time frame and the shift to the early integration of $\mathrm{PC}$ in the disease trajectory [57-61].

According to our definition of integrated PC, the timing of the initiation of PC care is not determined so that models that focused on either concurrent PC or end-of-life care were eligible. Despite the fact that, as mentioned above, end-of-life care in patients with non- 
malignant disease is difficult to realise in practice, this is not reflected in our results since representatives of both categories are included for patients with either malignant or non-malignant disease. As a consequence, our generic framework necessarily employs a diverse number of timings, ranging from concurrent to end-of-life. This is not without implications as offering a spectrum of options, over a uniquely defined one, implies that the final choice falls within the jurisdiction of the involved teams. In turn, this might lead to significantly diverse practices and, therefore, impede the benefits associated with PC. Future studies should thus focus on the convergence of opinions on the timing of PC so that ideally a standardized recommendation is crafted.

In the proposed generic framework, the involved team should be multidisciplinary in nature and involve experts from different fields. Functionality of multidisciplinary teams may be impeded by ambiguity of the distributed roles, conflicts between the team members, communication problems and issues related to leadership [62, 63]. However, extant studies in PC for patients with both malignant and non-malignant disease support the results of this study showing that multidisciplinary teams (MDTs) lead to better results when compared to unidisciplinary ones because they enhance continuity of care, evidence-based decision making, $\mathrm{ACP}$ and high quality care delivery [4, 64-66].

As the integration of $\mathrm{PC}$ requires bringing together specialists from different backgrounds and their wellorchestrated coordination, the development of protocols that quantify their collaboration is imperative for the successful implementation of PC. As mentioned in the Results section, the included studies employ miscellaneous collaboration strategies consisting of distinct components: collaboration between the team members (intra-team collaboration), multidisciplinary team meetings and protocols. The intra-team collaboration, which constitutes the only common component of all studies, is important because it assures the efficient implementation of the PC strategy. Further, for the aforementioned reasons multidisciplinary team meetings are also important for enhancing the continuity of care. Lastly, protocols have compelling advantages because they provide a clear guide for the next steps to be undertaken [67]. Since none of these components are redundant and our results show that they are complementary to each other in our generic framework, we propose to employ a strategy based on a combination of these.

Having addressed all the components of this generic framework, a comment on the implications that this framework has on the recommendations of the WHO [1] is in order. Overall, the proposed framework accords very well with the definition of PC provided by the
WHO, however, two important differences are identified. First, the proposed framework highlights the training of the healthcare providers, which is essential for the successful implementation of integrated PC; this is not the case of the WHO definition where training is absent. Second, although WHO recognizes the need for employing a team approach no explicit reference on multidisciplinarity is made. By contrast, the proposed framework emphasizes the importance of utilizing PC teams the members of which come from various backgrounds.

Finally, it is interesting to juxtapose the timings of PC. As mentioned above, due to the fact that the InSup-C definition of integrated PC does not comment on the timing, the proposed framework offers a multitude of timings; from concurrent to end-of-life. The definition of WHO states that PC "is applicable early in the course of illness, in conjunction with other therapies that are intended to prolong life...". However, as stated, this phrase does not explicitly promote early integration but rather on its range of applicability. In other words, the WHO definition does not narrow down the timing of PC to concurrent but rather expends to a spectrum of option in a manner similar to the proposed framework. Consequently, if attention has to be shifted to a concurrent timing, this should be explicitly reflected in the corresponding definitions.

Additional information may be drawn by comparing the five aspects of the generic framework against indicators for integrated PC. Such sets of indicators do exist in the literature, however, they correspond to $\mathrm{PC}$ in patients with cancer. Still, since cancer also pertains to the present such a comparison is fruitful. A recent systematic review provided a set of 38 indicators for integrated PC in oncology [68]. These indicators were grouped into five groups namely: structure of clinical programs, processes of clinical programs, education, research and administration. The same grouping was employed in the subsequent study [68], where a set of 43 indicators (13 major and 30 minor) was proposed based on a consensus of experts. Interestingly, with the exception of research, the generic framework, and the models that has been composed from, satisfies the majority of these indicators for both studies.

\section{Study limitations}

The first limitation of this study concerns the broadness of the topic under investigation. Integrated PC in cancer and non-cancer patients and without time specification is a very large area and requires a quite generic search strategy. Moreover, in view of this broadness, we were confronted with a substantially heterogeneous set of results that could only be accessed through a qualitative description. 
The search strategy employed was developed upon consensus between the PC experts involved in the project meetings. However, a unanimously agreed definition for integrated PC does not currently exist. Consequently, even though our strategy is broad, some evidence might have been missed due to the keywords used for the search strategy.

A third limitation concerns the choice of the languages of the included studies. The present study is confined to studies published in Dutch, English, French, German, Hungarian and Spanish, that were the languages that the project partners were knowledgeable of. It is, therefore, possible that studies published in other EU languages are available and not included [69].

Finally, we stress that the generic framework proposed herein has been based on models for patients with cancer and non-cancer disease. Its validity, therefore, is formally limited to these particular diseases. Although extrapolations to other types of life-threatening disease are tempting, further study in this direction is clearly needed. Also even though the proposed framework aims to be generic, one has to acknowledge limitations stemming from differences between healthcare systems, cultural and geographic factors and funding levels. Moreover, the preponderance of UK studies in this review should not go unnoticed. This is further supported by [70] who argue that generic models have to be flexible enough to be tailored to different settings; as a consequence, their recommendations should not be extremely stringent.

\section{Conclusions}

This is the first qualitative systematic literature review of models on integrated Palliative Care (PC) in patients with malignant and non-malignant disease in Europe. It represents the combined effort of an international consortium of six partners in six different European countries (Belgium, Germany, Hungary, Spain, The Netherlands and United Kingdom).

Based on the results, we have proposed a generic framework for integrated PC in patients with cancer and chronic disease. The proposed generic identifies the importance of employing a PC-trained multidisciplinary team and of having a threefold focus: on treatment, consulting and training. Each component of our framework has been already empirically assessed, however, the overall framework has not. Although one could extrapolate the existing empirical evidence in favour of our framework's efficacy, we believe that an empirical study for the framework as a whole should carried. Such a study not only will provide empirical evidence on the framework's efficacy but will also shed light on possible weaknesses that can be only traced postimplementation. Additionally, it would be interesting to conduct the same systematic review in non-European countries and performed a comparative analysis in a worldwide scale.

\section{Appendix}

In this appendix we present the search terms that were used for our search strategy.

"(hospices OR supportive care OR supportive care* OR end of life care* OR palliative* OR palliative care [MeSH Terms] OR hospice* OR terminal care* OR coordinated care $^{*}$ OR integrated care* OR transmural care* OR progressive patient care*) AND ("end stage disease" OR end stage disease* OR dying OR death [MeSH Terms] OR Chronic disease [MeSH Terms] OR Chronic disease* OR terminally ill* OR terminally ill [MeSH Terms] OR cancer) AND (care pathway* OR care pathway OR pathway* $O R$ patient transfer" OR patient transfer OR patient care team* OR managed care program* OR continuity of patient care OR patient care management $O R$ patient care plan* OR patient care planning OR illness trajectory $O R$ "advance care planning" OR advance care planning OR delivery of health care OR models of care OR model of care OR model organizational OR models organizational OR organizational model* OR guideline $\left.{ }^{*}\right)$ NOT ((birth) OR child) OR pediatrics)) NOT ((animals[mh] NOT humans[mh])) Filters: Publication date from 1995/01/01 to $2013 / 12 / 31 "$.

\section{Acknowledgements \\ "InSup-C, Patient-centered integrated palliative care pathways in advanced cancer and chronic disease", is funded by the European Union Seventh Framework Programme (FP7/HEALTH, under grant agreement 305555). InSup-C aims to identify the prerequisites for best practice in integrated palliative care with the overall objective of improving service delivery and service user outcomes. InSup-C is coordinated by Dr Jeroen Hasselaar of Radboud University Medical Center, Nijmegen, the Netherlands. Other partners are: University Hospital, Bonn, Germany; International Observatory on End-of- Life Care, Lancaster University, Lancaster and Sheffield University, United Kingdom; University of Pecs Medical School, Pecs, Hungary; University Hospital Leuven, Leuven, Belgium; World Health Organization, Geneva, Switzerland; European Association for Palliative Care (EAPC OnLus), Milan, Italy; Erasmus University, Rotterdam, The Netherlands; University of Navarra, Pamplona (Navarra), Spain; Mount Sinai Medical Center, New York, USA. We would like to specially thank Eva Dybek (University Hospital Bonn, Germany), Farina Hodiamont (University Hospital Bonn, Germany), Benjamin Ewert (University Hospital Bonn, Germany), Csilla Busa (University of Pécs Medical School, Hungary) and Zsuzsanna Kiss (University of Pécs Medical School, Hungary), Ildiko Radvanyi (University of Pécs Medical School, Hungary) and Juliano Ferreira Arcuri, for their assistance with collecting, screening and extracting the grey literature.}

\section{Funding}

"InSup-C, Patient-centered integrated palliative care pathways in advanced cancer and chronic disease", is funded by the European Union Seventh Framework Programme (FP7/HEALTH, under grant agreement 305555). InSup-C aims to identify the prerequisites for best practice in integrated palliative care with the overall objective of improving service delivery and service user outcomes. InSup-C is coordinated by Dr Jeroen Hasselaar of Radboud University Medical Center, Nijmegen, the Netherlands. Other partners are: University Hospital, Bonn, Germany; International Observatory on End-ofLife Care, Lancaster University, Lancaster and Sheffield University, United Kingdom; University of Pecs Medical School, Pecs, Hungary; University Hospital Leuven, Leuven, Belgium; World Health Organization, Geneva, Switzerland; European Association for Palliative Care (EAPC OnLus), Milan, Italy; Erasmus University, Rotterdam, The Netherlands; University of Navarra, Pamplona (Navarra), Spain; Mount Sinai Medical Center, New York, USA. 


\section{Availability of data and materials}

The authors will make the data available on the InSup-C website (http:// www.insup-c.eu/). The data is also available from the authors on request.

\section{Authors' contributions}

NS was the primary reviewer of this systematic review. She also contributed to the design, selection, collection, analysis and interpretation of the study's data including those retrieved from the grey literature. Additionally, she was the writer of this manuscript. KVB was the secondary reviewer of this systematic review. She also contributed to the design, selection, collection, analysis and interpretation of the study's data including those retrieved from the grey literature and she critically revised the manuscript. NP and JGH were the principal reviewers of the manuscript throughout from the early to the last drafts. SP, LR, CC, MG and AC have been involved in revising critically the manuscript for important intellectual content. SH, EG, and MEvdE contributed to the selection, collection, analysis and interpretation of the study's data retrieved from the grey literature. They have also contributed in the revision of the manuscript by providing critical and valuable remarks. JM was the main coordinator of this study. He has been also involved in the revision of the manuscript for important intellectual content. All the authors participated in InSup-C meetings by providing valuable insight for the different stages of the study and they have given their final approval of the version to be published.

\section{Competing interests}

The authors declare that they have no competing interests.

\section{Consent for publication}

Not applicable.

\section{Ethics approval and consent to participate}

Ethical approval was not required for this study. However this study was performed according to the standards of PRISMA guidelines. No consent was required for the conduction of this study.

\section{Author details}

${ }^{1}$ Department of Radiation-Oncology and Palliative Medicine, University Hospital Gasthuisberg, Herestraat 49, 3000 Leuven, Belgium. ${ }^{2}$ Department of Anesthesiology, Pain and Palliative Medicine, Radboud University Nijmegen Medical Centre, Nijmegen, The Netherlands. ${ }^{3}$ International Observatory on End of Life Care, Division of Health Research, Lancaster University, Lancaster, UK. ${ }^{4}$ Atlantes, ICS, University of Navarra, Pamplona, Spain. ${ }^{5}$ Faculty of Medicine, Institute of Family Medicine, University of Pécs Medical School, Pécs, Hungary. ${ }^{6}$ Department of Palliative Medicine, University Hospital Bonn, Bonn, Germany. ${ }^{7}$ IdiSNA, Instituto de Investigación Sanitaria de Navarra, Pamplona, Spain.

\section{Received: 23 February 2016 Accepted: 30 June 2016}

Published online: 08 July 2016

\section{References}

1. World Health Organization. World Health Organization Definition of Palliative Care. http://www.who.int/cancer/palliative/definition/en/ Accessed 23 March 2012

2. Radbruch L, Payne S. White Paper on standards and norms for hospice and palliative care in Europe: part 1 Recommendations from the European Association for Palliative Care. Eur J Palliat Care. 2009;16(6):278-89.

3. Council of Europe. Recommendation Rec (2003) 24 of the Committee of Ministers to member states on the organisation of palliative care. http:// www.eapcnet.eu/LinkClick.aspx?fileticket=3KJ5U3BQLVY\%3d\&tabid=1709 Accessed 6 November 2012.

4. Temel JS, Greer JA, Muzikansky A, Gallagher ER, Admane S, Jackson VA, Dahlin CM, Blinderman CD, Jacobsen J, Pirl WF et al. Early palliative care for patients with metastatic non-small-cell lung cancer. N Engl J Med. 2010; 14(8):733-42.

5. Bakitas MA, Tosteson TD, Li Z, et al. Early versus delayed initiation of concurrent palliative oncology care: patient outcomes in the ENABLE III randomized controlled trial. J Clin Oncol. 2015;33(13):1438-45.

6. Chan R, Webster J. End-of-life care pathways for improving outcomes in caring for the dying. Cochrane Database Syst Rev. 2013;1:1-16.
7. Lo S, Chan C, Sze W, Yuen K, Wong C, Ng T, Tung Y. The implementation of an end-of-life integrated care pathway in a Chinese population. Int J PalliatNurs. 2009;15:384-8.

8. Bookbinder M, Blank AE, Arney $E$, Wollner $D$, Lesage $P$, McHugh $M$, Indelicato RA, Harding S, Barenboim A, Mirozyev T, Portenoy RK. Improving end-of-life care: development and pilot-test of a clinical pathway. J Pain Symptom Manage. 2005;29(6):529-43.

9. Partridge A, Seah D, King T, Leighl NB, Hauke R, Von Roenn JH WD. Developing a service model that integrates palliative care throughout cancer care: the time is now. J Clin Oncol. 2014;32:3330.

10. Bruera E, Sweeney C. Palliative care models: international perspective. J Palliat Med. 2002;5(2):319-27.

11. Bakitas M, Lyons KD, Hegel MT, et al. Effects of a palliative care intervention on clinical outcomes in patients with advanced cancer: the Project ENABLE II randomized controlled trial. JAMA. 2009;302:741-9.

12. Thomas K. Prognostic indicators guidance (PIG). 4th edn. The Gold Standards Framework Centre in End of Life Care; 2011. Available from: http://www.goldstandardsframework.org.uk/cd — content/uploads/files/ General\%20Files/Prognostic\%20Indicator\%20Guidance\%200ctober\%202011.pdf. Accessed July 2016.

13. Boyd K, Murray SA. Recognising and managing key transitions in end of life care. BMJ. 2010;341:c4863.

14. Gómez-Batiste X, Martínez Muñoz M, Blay C, Espinosa J, Contel JC, Ledesma A. Identifying needsand improving palliative care of chronically ill patients: a community-oriented, population-based, public-health approach. Curr Opin Support Palliat Care. 2012;6:371-8.

15. Gómez-Batiste X, Martínez-Muñoz M, Blay C, Amblàs J, Vila L, Costa X, et al. Identifying chronic advanced patients in need of palliative measures and health and social care services in the general population: development of the NECPAL tool and preliminary findings of the NECPAL prevalence study in Catalonia. BMJ Support Palliat Care. 2012;0:1-9.

16. Calman K, Hine D. A policy framework for commissioning cancer services. A report by the Expert Advisory Group on Cancer to the Chief Medical Officers of England and Wales. London: Department of Health; 1995.

17. Hawker S, Payne S, Kerr C, Hardey M, Powell J. Appraising the evidence: reviewing disparate data systematically. Qual Health Res. 2002;12(9):1284-99.

18. Oishi A, Murtagh FE. The challenges of uncertainty and interprofessional collaboration in palliative care for non-cancer patients in the community: a systematic review of views from patients, carers and health-care professionals. Palliat Med. 2014;28:1081-98.

19. Rigby J, Payne S, Froggatt K. What evidence is there about the specific environmental needs of older people who are near the end of life and are cared for in hospices or similar institutions? A literature review. Palliat Med. 2010;24(3):268-85.

20. Jordhøy MS, Fayers P, Loge JH, Ahlner- Elmqvist M, Kaasa S. Quality of life in palliative cancer care: results from a cluster randomized trial. J Clin Oncol. 2001;19:3884-94.

21. Smeenk FW, de Witte LP, van Haastregt JC, Schipper RM, Biezemans HP, Crebolder HF. Transmural care. A new approach in the care for terminal cancer patients: its effects on re-hospitalization and quality of life. Patient Educ Couns. 1998;35:189-99.

22. Colombet I, Montheil V, Duran JP, Gillaizeau F, Niarra R, et al. Effect of integrated palliative care on the quality of end-of-life care: retrospective analysis of 521 cancer patients. BMJ Support Palliat Care. 2012;2:239-47.

23. Schreml W, Staub JP. Integrated palliative care within a general hospital. Support Care Cancer. 2000;8:80-3.

24. Constantini M, Romoli V, Di Leo S, et al. Liverpool care pathway for patients with cancer in hospital: a cluster randomised trial. Lancet. 2014;383:226-37.

25. Veerbeek L, van Zuylen L, Swart SJ, van der Maas PJ, de Vogel-Voogt E, van der Rijt CC, van der Heide A. The effect of the Liverpool Care Pathway for the dying: a multi-centrestudy. Palliat Med. 2008;22(2):145-51.

26. Grande GE, Todd CJ, Barclay SIG, Farquhar MC. A randomized controlled trial of a hospital at home service for the terminally ill. Palliat Med. 2000;14: 375-85.

27. Vicente C, Marco J, Plaza S, Flox G. Influencia del Plan Integral de CuidadosPaliativos de la Comunidad de Madrid en la actividad de unaunidad de cuidadospaliativoshospitalaria. Med Palativa. 2010;20(2): 60-3.

28. Sampson EL, Jones L, Thune-Boyle ICV. Palliative assessment and advance care planning in severe dementia: an exploratory randomized controlled trial of a complex intervention. Palliat Med. 2011;25:197-209. 
29. Higginson IJ, McCrone P, Hart SR, Burman R, Silber E, Edmonds PM. Is shortterm palliative care cost-effective in multiple sclerosis? A randomized phase II trial. J Pain Symptom Manage. 2009;38:816-26.

30. Edmonds P, Hart S, Gao W, Vivat B, Burman R, Silber E, Higginson IJ. Palliative care for people severely affected by multiple sclerosis: evaluation of a novel palliative care service. MultScler. 2010;16(5):627-36.

31. Koffman J, Higginson I, Naysmith A. Hospice at home-a new service for patients with advanced HIV/AIDS: a pilot evaluation of referrals and outcomes. Br J Gen Pract. 1996;46:539-40.

32. Pattenden J, Mason AR, Lewin RJP. Collaborative palliative care for advanced heart failure: outcomes and costs from the 'Better Together' pilot study. BMJ Support Palliat Care. 2013;3:69-76.

33. Navarro R, Valls M, Castellano E. Atención a pacientescrónicosavanzados no oncológicos con necesidad de cuidados al final de la vida en un hospital de media y larga estancia. Med Paliativa. 2011;18(2):54-62.

34. Fayers $\mathrm{P}$, Aaronson N, Bjordal $\mathrm{K}$, on behalf of the EORTC study group, et al. EORTC QLQ-C30 Scoring Manual. Brussels: EORTC Study Group on Quality of Life; 1995.

35. Rossier P, Wade DT. The Guy's Neurological Disability Scale in patients with multiple sclerosis: a clinical evaluation of its reliability and validity. Clin Rehabil. 2002;16(1):75e95.

36. Rossier P, Wade DT. Validity and reliability comparison of 4 mobility measures in patients presenting with neurologic impairment. Arch Phys Med Rehabil. 2001;82(1):9e13.

37. Hearn J, Higginson IJ. Development and validation of a core outcome measure for palliative care: the palliative care outcome scale. Qual Health Care. 1999;8:219e227.

38. Higginson I, McCarthy M. Validity of the support team assessment schedule: do staffs ratings reflect those made by patients or their families? Palliat Med. 1993;7:219-28.

39. Abbey J, Piller N, De BA, Esterman A, Parker D, Giles L, et al. The Abbey pain scale: a 1-min numerical indicator for people with end-stage dementia. Int J Palliat Nurs. 2004;10:6-13.

40. Fuchs-Lacelle $\mathrm{S}$, Hadjistavropoulos T. Development and preliminary validation of the pain assessment checklist for seniors with limited ability to communicate (PACSLAC). Pain Manag Nurs. 2004;5:37-49.

41. Lefebvre-Chapiro S. The Doloplus scale - evaluating pain in the elderly. Eur J Palliat Care. 2001:8:191-3.

42. Costantini M, Beccaro M, Merlo F. The last three months of life of Italian cancer patients. Methods, sample characteristics and response rate of the Italian Survey of the Dying of Cancer (ISDOC). Palliat Med. 2005;19(8):628-38.

43. Bedard M, Molloy DW, Squire L, et al. The Zarit Burden Interview: a new short version and screening version. Gerontologist. 2001;41(5):652e657.

44. Briere J, Elliott DM. Clinical utility of the impact of event scale: psychometrics in the general population. Assessment. 1998;5:171-80.

45. Ellershaw J, Ward C. Care of the dying patient: the last hours or days of life. BMJ. 2003;326:30-4.

46. Neuberger J. More Care Less Pathway: A Review of the Liverpool Care Pathway. Crown Copyright; 2013. Available from: https://www.gov.uk/ government/uploads/system/uploads/attachment_data/file/212450/ Liverpool_Care_Pathway.pdf. Accessed July 2016.

47. Hughes S, Preston N, Payne S. What went wrong with the Liverpool Care Pathway and how can we avoid making the same mistakes again? Int J Palliat Nurs. 2013;19(8):372-3.

48. McConnell T, O'Halloran P, Donnelly M, Porter S. Factors affecting the successful implementation and sustainability of the Liverpool Care Pathway for dying patients: a realist evaluation. BMJ Support Palliat Care. 2015;5(1):70-7.

49. Gibbins J, McCoubrie R, Forbes K. Why are newly qualified doctors unprepared to care for patients at the end of life? Med Educ. 2011;45:389-99.

50. Bowden J, Dempsey K, Boyd K, Fallon M, Murray SA. Are newly qualified doctors prepared to provide supportive and end-of-life care? A survey of foundation year 1 doctors and consultants. J R Coll Physicians Edinb. 2013;43:24-8.

51. The Lancet. End-of-life care: the neglected core business of medicine. Lancet. 2012;379:1171.

52. Field M, Cassel C. Approaching death Improving care at the end of life. Washington, DC: National Academy Press; 1997.

53. Murray SA, Kendall M, Boyd K, Sheikh A. Illness trajectories and palliative care. BMJ. 2005:330:1007-11.

54. Christakis NA, Lamont EB. Extent and determinants of error in doctors' prognoses in terminally ill patients: prospective cohort study. BMJ. 2000; 320:469-72.
55. Zambroski $\mathrm{CH}$. Managing beyond an uncertain illness trajectory: palliative care in advanced heart failure. Int J Palliat Nurs. 2006;12(12):566-73.

56. Jaarsma $T$, Beattie JM, Ryder $M$, et al. Palliative care in heart failure: a position statement from the palliative care workshop of the Heart Failure Association of the European Society of Cardiology. Eur I Heart Fail. 2009;11:433-43.

57. Kendall M, Boyd K, Campbell C, Cormie P, Fife S, Thomas K, Weller D, Murray SA. How do people with cancer wish to be cared for in primary care? Serial discussion groups of patients and carers. Fam Pract. 2006;23(6):644-50.

58. Harding R, Selman L, Agupio G, Dinat N, Downing J, Gwyther L, Mashao T, Mmoledi K, Sebuyira LM, Ikin B, Higginson IJ. The prevalence and burden of symptoms amongst cancer patients attending palliative care in two african countries. Eur J Cancer. 2011;47(1):51-6.

59. Rowland K, Schumann S-A. Palliative care: earlier is better. J Fam Pract. 2010; 59:695-8.

60. Gaertner J, Wolf J, Frechen S, et al. Recommending early integration of palliative care-does it work? Support Care Cancer. 2012;20:507-13.

61. Cummings I. The interdisciplinary team. In: Doyle D, Hanks GW, MacDonald $\mathrm{N}$, editors. Oxford textbook of palliative medicine. Oxford: Oxford University Press; 1998.

62. Street A, Blackford J. Communication issues for the interdisciplinary community palliative care team. J Clin Nurs. 2001;10:643-50.

63. Finlay IG, Higginson IJ, Goodwin DM, et al. Palliative care in hospital, hospice, at home: results from a systematic review. Ann Oncol. 2002;13 Suppl 4:257-64.

64. Hearn J, Higginson IJ. Do specialist palliative care teams improve outcomes for cancer patients? A systematic literature review. Palliat Med. 1998;12:317-32.

65. Crawford GB, Price SD. Team working: palliative care as a model of interdisciplinary practice. Med J Aust. 2003;179:S32-4.

66. Detering KM, Hancock AD, Reade MC, et al. The impact of advance care planning on end of life care in elderly patients: Randomized controlled trial. BMJ. 2010;340:c1345.

67. Curtis JR, Rubenfeld GD. Improving palliative care for patients in the intensive care unit. J Palliat Med. 2005;8:840-54.

68. Hui D, Kim YJ, Park JC, et al. Integration of oncology and palliative care: a systematic review. Oncologist. 2015;20:77-83.

69. Centeno C, Lynch T, Donea O, Rocafort J, Clark D. EAPC Atlas of Palliative Care in Europe 2013. Fullth ed. Milan: EAPC Press; 2013.

70. Hui D, Bruera E. Models of integration of oncology and palliative care. Ann Palliat Med. 2015;4:89-98.

\section{Submit your next manuscript to BioMed Central and we will help you at every step:}

- We accept pre-submission inquiries

- Our selector tool helps you to find the most relevant journal

- We provide round the clock customer support

- Convenient online submission

- Thorough peer review

- Inclusion in PubMed and all major indexing services

- Maximum visibility for your research

Submit your manuscript at www.biomedcentral.com/submit
Biomed Central 